\title{
Nailing versus plating in the management of proximal extra-articular tibial fractures: a systematic review
}

\begin{abstract}
Background: Problem both intramedullary nailing (IMN) and plating have pearls and pitfalls in treating proximal tibial extra-articular fractures. The current study sought to provide clinical evidence for comparison of the two methods in treating such injury.

Methods: A systematic search for articles published from January, 1990 to September, 2016, using PubMed, Embase and the Cochrane library was conducted. Clinical researches comparing the outcomes of nailing and plating for proximal extra-articular tibial fractures, on human subjects over 18years old were included. No limit of language and publication status was applied. The quality of the included randomized controlled studies was analyzed with Cochrane Collaboration Risk of Bias Tool. And the non-randomized studies were assessed using the Risk of Bias Assessment Tool for Non-Randomized Studies (RoBANS).

Results: Out of 299 preliminary search results, one randomized controlled trial and one retrospective cohort study were included in the current systematic review of 100 cases (44 in the RCT and 56 in the retrospective study, 41 IMN cases and 59 plate cases).

Interpretation: The meta-analysis of the current study found similar union rate, mal-union rate and infection rate for IMN and plate. IMN was more patient satisfactory, but at the same time, more technically requiring. However, the final conclusion that IMN and plate had the same outcome in treating proximal tibial extra-articular fractures should still be made with caution, because of the relatively small sample size of this systematic review.
\end{abstract}

Keywords: proximal extra-articular tibial fracture, intramedullary nail, plate, mal-union, infection
Volume 7 Issue 4 - 2017

\author{
Shiqiang Cen, Xi Liu \\ Department of Orthopaedic Surgery Sichuan University, China
}

Correspondence: Shiqiang Cen Department of Orthopaedic Surgery Sichuan University West China Hospital No 37 Guo Xue Avenue Chengdu Sichuan Province, China,Tel +8618980601849, Email ahsirsj7201@|26.com

Received: April 06, 2017 | Published: June 09, 2017
Abbreviations: RoBANS, risk of bias assessment tool for non-randomized studies; MIN, intramedullary nailing; LISS, less invasive stabilization system; PRISMA, preferred reporting items for systematic reviews and meta-analysis; WHO, world health organization; ICTRP, international clinical trials registry platform; ISRCTN, international standard randomized controlled trial number; OR, odds ratio; SPSS, statistical package for the social sciences

\section{Introduction}

Tibia is one of the most common sites for fractures to take place. Proximal tibial extra-articular fractures commonly refer to those involving the proximal one third of the bone, with extension into the metaphyseal region. The anatomical structure and position of this part makes its treatment challenging. The relatively short proximal segment, with widened intramedullary canal and soft cancellous bone texture, provides less mechanical restrain for reduction maneuver and internal fixation than the shaft does. ${ }^{1}$ And displacing stress from adjacent structures like the proximal tibia-fibular syndesmosis, the patellar tendon and surrounding muscles may lead to varus and apex anterior angulation malalignment. ${ }^{2}$ Early studies suggested that intramedullary nailing (IMN) was not the best choice for this kind of fracture for the high risk of mul-reduction and progressive malunion and that its use should be limited to alternative for plates and external fixation. ${ }^{1,3}$ However, recent studies show that this problem can be significantly improved with modifications to nail design and multiple surgical techniques including wise selection of nail starting point, supra-patellar nail insertion, nailing in semi-extended position, use of femoral distractor, poller/blocking screws and supplemental unicortical plates and et al., ${ }^{4,5}$ Before the improvements mentioned above came into being, plating was considered the best choice for proximal tibial fractures. Nevertheless, plating needed more invasive dissection and tissue stripping than nailing. And due to the anatomical characteristics of proximal tibia, whose medial side is lack of muscle coverage, the morbidity associated with deep surgical site infection in plated proximal tibial fractures was rather high. ${ }^{6}$ But the development of low profile plates and LISS (less invasive stabilization system) technique made the situation better. ${ }^{7-9}$ Therefore, currently, both ways can achieve excellent results for proximal tibial fractures. The current systematic review sought to gather clinical evidence for comparison of nailing with plating for the treatment of proximal extra-articular tibial fractures in terms of radiological and clinical outcomes as well as their post-operative complication profiles.

\section{Material and methods}

\section{Search strategy}

The current systematic review was carried out according to the Cochrane Handbook for Systematic Reviews of Interventions and the Preferred Reporting Items for Systematic Reviews and MetaAnalysis (PRISMA) statement. The PubMed-Medline database of the United States National Library of Medicine, Embase, and the Cochrane library were electronically searched for articles published from January, 1990 to September, 2016. Ongoing clinical studies were searched in three databases including "clinicaltrials" at https:// clinicaltrials.gov, World Health Organization (WHO) International Clinical Trials Registry Platform (ICTRP) at http://apps.who.int/ trialsearch, and the International Standard Randomized Controlled Trial Number (ISRCTN) at http://www.isrctn.com. 
Standard Randomized Controlled Trial Number (ISRCTN). The clinical question in "PICO" format (P: patient problem / population; I: Intervention; C: Comparison; O: Outcome) in the present study was: In adult patients with proximal one third extra-articular tibial fracture, $\mathrm{AO} 41 \mathrm{~A}$ (based on $\mathrm{AO}$ classification of fractures) ${ }^{10}$ do nailing compared with plating have similar union rate, malunion rate, union time, time to full weight-bearing, range of motion of the knee, operative time, infection rate, rate of knee pain, and re-operation rate?

A systematic search was conducted in Cochrane Lib, PubMed and Embase for all relevant articles published from January 1960 to September 2016. No limits were applied in terms of language or publication status. Authors of the latest and unpublished studies were contacted for details concerning their ongoing work. Study selection, data extraction and quality assessment were conducted by two independent reviewers working simultaneously. When inter-observer disagreements took place, a third reviewer was available to settle them. The following searching strategy was adopted:

a. (("fracture* of tibia") OR "tibial fracture*") OR "Tibial Fractures"Mesh-13793

b. "Fracture Fixation, Intramedullary"Mesh OR "intramedullary nail*”-9291

c. "Bone Plates"Mesh OR "plate" OR "plating" OR LISS OR LCP OR LC-DCP or MIPO-113703

d. 1 AND (2 AND 3)-292

e. Filter with human-266

\section{Inclusion and exclusion criteria}

Before starting the search, the inclusion and exclusion criteria adopted were established. The inclusion criteria were mainly original clinical researches comparing the outcomes of nailing and plating for proximal extra-articular tibial fractures, on human subjects over 18years old only, with the following study designs:
a. Randomized controlled trials
b. Prospective cohort studies
c. Retrospective cohort studies
d. Case control studies
e. Cross-over studies

\section{The exclusion criteria were as following}

i. Case reports

ii. Case series without control group

iii. Surgical technique reports

iv. Review articles, including other systematic reviews and metaanalyses

v. Studies reporting the clinical results of nailing or plating for proximal extra-articular tibial fractures respectively without comparison

vi. Experimental laboratory studies

vii. Biomechanical studies on corpses or animals

viii. Finite element analyses

ix. Letters, correspondences or comments

x. Studies discussing non-unions instead of fresh fractures

xi. Studies published before 1990, for both the nail design and surgical technique of early nailing surgeries were not as good as their modern counterparts

xii. Ongoing studies in its early stage with no useful data available

\section{Risk of bias assessment and data extraction}

All included randomized controlled studies were analyzed with Cochrane Collaboration Risk of Bias Tool for risk of bias and methodological quality following the principles of the Cochrane Handbook for Systematic Reviews of Interventions, by evaluating the way of random allocation, presence and quality of allocation concealment and blinding, as well as existence of incomplete outcome data and selective outcome reporting ${ }^{11-13}$ and the non-randomized studies were assessed using the Risk of Bias Assessment Tool for Non-Randomized Studies (RoBANS). After the final agreement has been made on the articles included for study, the following data were retrieved and extracted from each article: Name of the first author; publication year (unpublished researches are simply marked as "ongoing"); patients' demographic status including gender and age; the entire sample size and the patients receiving nails and plates respectively; average time of follow-up; union rate; mal-union rate; union time; time to full weight-bearing; range of motion of the knee; operative time; infection rate; rate of knee pain; and re-operation rate (Table $1 \& 2$ ).

Table I Risk of bias assessment of the RCT

\begin{tabular}{llllll}
\hline $\begin{array}{l}\text { Adequate } \\
\text { Randomization Method }\end{array}$ & $\begin{array}{l}\text { Allocation } \\
\text { Concealment }\end{array}$ & Blinding & Incomplete Outcome & \multicolumn{2}{c}{$\begin{array}{l}\text { Free of Selective } \\
\text { Reporting }\end{array}$} \\
\hline Meena RC, 2015 & Yes & Yes & Unclear (Only to the nurse) & Yes & Yes \\
\hline
\end{tabular}

Table 2 Risk of bias assessment of the retrospective cohort study

\begin{tabular}{|c|c|c|c|c|c|c|c|}
\hline & $\begin{array}{l}\text { Selection of } \\
\text { Participants }\end{array}$ & $\begin{array}{l}\text { Confounding } \\
\text { Variables }\end{array}$ & $\begin{array}{l}\text { Measurement } \\
\text { of Intervention } \\
\text { (Exposure) }\end{array}$ & $\begin{array}{l}\text { Blinding for } \\
\text { Outcome } \\
\text { Assessment }\end{array}$ & Incomplete & $\begin{array}{l}\text { Selective } \\
\text { Outcome } \\
\text { Reporting }\end{array}$ & Free of \\
\hline & & & & & \multicolumn{2}{|c|}{ Outcome Data } & other Bias \\
\hline Lindvall E, 2009 & High quality & Low quality & High quality & Low quality & High quality & High quality & Yes \\
\hline
\end{tabular}

\section{Statistical analysis}

Dichotomous data were analyzed with odds ratio (OR), while standard mean difference with $95 \%$ confidence interval was calculated for continuous data. A data collection table designed beforehand was prepared for all the data extracted. The statistical analysis was performed by an independent statistician using the Statistical Package for the Social Sciences (SPSS version 19.0; SPSS, Chicago, IL, USA). 


\section{Results}

The initial search retrieved 266 records from PubMed, 178 from Embase and 29 from Cochrane lib. After ruling out duplicate records by importing the search results into Endnote software version 7, 299 records were left. 256 records were excluded after initial title and abstract evaluation, leaving 43 for full-text evaluation. Finally, one RCT and one retrospective cohort study were included for analysis (Figure 1). An ongoing multicenter randomized trial comparing IM nails and plate fixation (IMPRESS) was found on the website of clinical trials. The authors were contacted for the current progress of the study. Unfortunately, this study was still in the stage of case enrollment, and no data was available for the moment.

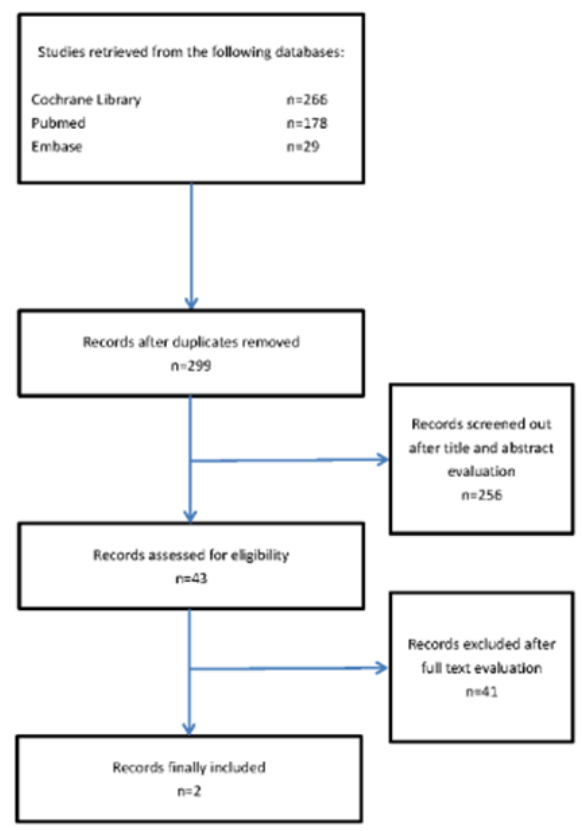

Figure I PRISMA flowchart showing the literature management process of the current review.

\section{Study characteristics}

The RCT included was conducted on 58 skeletally mature closed extra-articular proximal tibial fracture (OTA 41-A2/A3) patients who were randomized to either minimally invasive proximal tibial plating or IMN, but 14 patients were excluded because of loss of follow-up, leaving only 44 patients for the final outcome analysis after 1year's follow-up. Outcome measures including operating time, hospital stay, infection rate, fracture union time, angulation of the fracture, and the knee range of motion were compared between the two groups. The retrospective cohort study accounted for 56 cases for analysis, including $22 \mathrm{IMN}$ and 34 percutaneous locked plate cases, all followed up for at least lyear. Final outcomes were assessed by comparing rates of union, malunion, malreduction (defined as .5degrees angulation in any plane), infection, and removal of implants between the two groups.

\section{Study quality}

According to the Cochrane Collaboration's tool for assessing risk of bias, the included randomized study was assessed to be at unclear risk of bias because the blinding of participants, personnel and outcome assessors was not clarified. However, there was low risk of bias in randomization and allocation concealment, and was free from Incomplete outcome, selective reporting, and other forms of bias. There was no conflict of interest in this study. The retrospective cohort study was assessed as having high risk of bias for the low quality in confounding variables and blinding, although the quality in other domains including participants selection, Measurement of intervention, and ruling out incomplete Outcome data and selective outcome reporting was high.

\section{Radiologic outcomes}

Both studies evaluated the patients' fracture alignment and union rate radiographically. The RCT concluded that both IMN and plate patients had a slight varus and procurvatum inclination immediately after operation, but there was no statistical difference between the two groups. And secondary loss of reduction was seen in neither group, confirming the stability of both methods and resulting in similar mal-union rate in the two groups. Both groups had a high union rate ( $94.7 \%$ for IMN and $96 \%$ for plate) with no statistical inter-group difference, but the union time for IMN was significantly shorter than that for plate. While in the retrospective study, the risk of procurvatum malreduction deformity with IMN was found to be twice of that of plate. However, pooled results from the two studies failed to find any statistical difference between IMN and plate in union rate (Figure 2) as well as in mal-alignment (Figure 3).

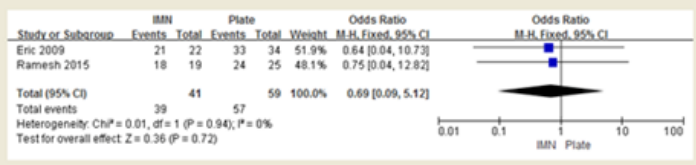

Figure $2 \mathrm{~A}$ forest plot showing pooled data of union rates of IMN and plate.

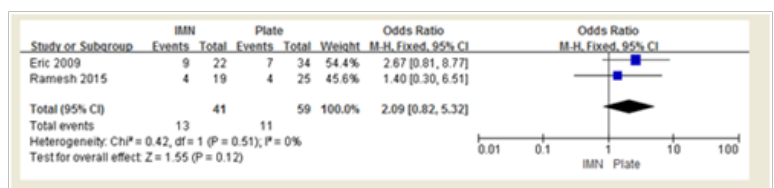

Figure $3 \mathrm{~A}$ forest plot showing pooled data of mal-union rates of IMN and plate.

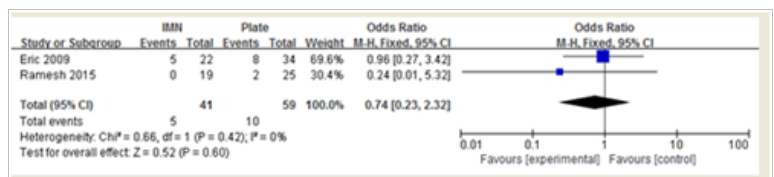

Figure $4 \mathrm{~A}$ forest plot showing pooled data of infection rates of IMN and plate.

\section{Functional outcomes}

The RCT found no clear difference in knee range of motion between IMN and plate, while the other study didn't mention this part at all.

\section{Complications}

The RCT found no clear advantage of either technique in infection rate, with $0 \%$ of infection in the IMN group and $8 \%$ in the plate group. In the retrospective study, the overall infection rate was similar in IMN and plate groups ( $23 \%$ and $24 \%$ respectively), but there were a higher percentage of open fractures in the IMN group. Further analysis according to open and closed fractures showed that the two 
techniques had the same infection rate $(33 \%)$ for open fractures. And pooled results, again, found no statistical difference in infection rate between the two methods (Figure 4). Rate of implant removal because of discomfort or pain after plating was 3times higher than that after IMN fixation.

\section{Other terms}

The RCT reported significant shorter postoperative hospital stay, time to full weight bearing, and union time for IMN fixation. The retrospective study revealed that IMN fixation needed additional surgical techniques like blocking screws more frequently than plate fixation.

\section{Discussion}

As mentioned above, it has been a dilemma for orthopedic surgeons to decide whether to choose IMN or plate for the fixation of proximal tibial extra-articular fractures, for they have both been used for a long time, and good as well as bad results have been reported for both of them. Early in 1990s, IMN was once thought to be unfit for proximal tibial fractures, mostly because of the unacceptably high rate of malalignment, usually apex angulation, after surgery. However, IMN was still not given up and more studies about its use in the treatment of this troublesome fracture type still kept appearing, probably because of the potential biomechanical advantage over other fixation methods, like plates and external fixators, which were eccentric in nature, and the relatively less need for soft tissue stripping. In 2003, a systematic review published by the Evidence-Based Orthopaedic Trauma Working Group concluded that IMN could reduce the risk of infection, but had higher rate of malunion than plates. ${ }^{14}$ And several non-systematic reviews held similar views. ${ }^{2,15}$

However, according to the result of the current review, no statistically significant difference could be found in union rate, malunion rate, knee function and infection rate between IMN and plate. The differences between the two ways of fixation are that IMN has shorter postoperative hospital stay, time to full weight-bearing and union but requires additional surgical techniques like blocking screws, and that plate has a three times higher symptomatic removal rate. It seemed as if this could be put in short that IMN and plate had similar final outcomes, and that IMN was more patient satisfactory but, at the same time, more technically requiring. We believe that this contradiction was due to several aspects. Firstly, the systematic review published in 2003 included only case series which merely provided Grade C clinical evidence. Lack of comparable control group with identical or at least similar baseline data, the IMN and plate groups in this study could have different average age, gender ratio, injury severity and et al, and therefore, the result of inter-group comparison could be misleading. ${ }^{11}$ This could be the reason for the highest implant failure rate for IMN compared with plate and even with external fixator in this systematic review, which was hard to explain, for according to most of the biomechanical studies, IMN has the strongest stability. ${ }^{16-18}$

Secondly, IMN and plate fixation techniques and instruments for proximal tibial fractures themselves are evolving. As is known, the LISS technique has obvious advantage over conventional plate fixation in tissue protection and therefore can reduce the risk of soft tissue complications. But it was not introduced by Synthes until 1990, and its early clinical result was reported around 2000. ${ }^{19-21}$ Some of the cases fixed with plates included in the previous systematic review at that time didn't adopt this technique, while in the current systematic review, all the plate fixations were performed using LISS system. Meanwhile, techniques for IMN fixation of proximal tibial fractures at that time were far less developed. Only one study included in that systematic review used blocking screws. Other techniques like supra-patellar nail insertion, nailing in semi-extended position, use of femoral distractor, and supplemental unicortical plates or external fixators were not mentioned at all. Some studies were even still using unreamed small diameter nails. ${ }^{22}$ With the two techniques both developing with time, their shortcomings became less obvious, making both of them more acceptable, and hence the gap between them narrowed.

However, the final conclusion that IMN and plate have the same outcome in fixation of proximal tibial extra-articular fractures should still be made with caution, because of the relatively small sample size of this systematic review (only one single centered RCT with 58 cases and a retrospective cohort study with 44 cases were defined), and therefore resulting limited power of test. And this was due to the lack of relevant high quality clinical evidence that presently existed, especially large scale multi-centered randomized controlled studies. And the answer to the question may possibly change after the result of IMPRESS has been published in the near future.

\section{Acknowledgments}

None.

\section{Conflicts of interest}

Author declares there are no conflicts of interest.

\section{Funding}

None.

\section{References}

1. Lang GJ, Cohen BE, Bosse MJ, et al. Proximal third tibial shaft fractures. Should they be nailed? Clin Orthop Relat Res. 1995;315:64-74.

2. Krieg JC. Proximal tibial fractures: current treatment, results, and problems. Injury. 2003;34(Suppl 1):A2-A10.

3. Safran O, Liebergall M, Segal D, et al. Proximal tibial fractures--should we nail them? Am J Orthop (Belle Mead NJ). 2001;30(9):681-684.

4. Stinner DJ, Mir H. Techniques for intramedullary nailing of proximal tibia fractures. Orthop Clin North Am. 2014;45(1):33-45.

5. Tejwani N, Polonet D, Wolinsky PR. Controversies in the intramedullary nailing of proximal and distal tibia fractures. J Am Acad Orthop Surg. 2014;22(10):665-673.

6. Parkkinen M, Madanat R, Lindahl J, et al. Risk Factors for Deep Infection Following Plate Fixation of Proximal Tibial Fractures. J Bone Joint Surg Am. 2016;98(15):1292-1297.

7. Stannard JP, Finkemeier CG, Lee J, et al. Utilization of the less-invasive stabilization system internal fixator for open fractures of the proximal tibia: a multi-center evaluation. Indian J Orthop. 2008;42(4):426-430.

8. Tong DK, Ji F, Cai XB. Locking internal fixator with minimally invasive plate osteosynthesis for the proximal and distal tibial fractures. Chin J Traumatol. 2011;14(4):233-236.

9. Devkota P, Manandhar HK, Khadka PB, et al. Less invasive stabilization system for the management of proximal tibia fractures. Eur J Orthop Surg Traumatol. 2003;24(6):993-998.

10. Müller MENS, Koch P, Schatzker J. The comprehensive classification of fractures of long bones. 1st. ed. Springer-Verlag, Berlin, Heidelberg, New Yorkm, USA. 1990. 
11. Liberati A, Altman DG, Tetzlaff J, et al. The PRISMA statement for reporting systematic reviews and meta-analyses of studies that evaluate health care interventions: explanation and elaboration. $B M J$. 2009;339:b2700.

12. Higgins JP, Altman DG, Gøtzsche PC, et al. Cochrane Bias Methods Group; Cochrane Statistical Methods Group. The Cochrane Collaboration's tool for assessing risk of bias in randomised trials. $B M J$ . 2011;343:d5928.

13. Julian PT Higgins, Sally Green. Cochrane Handbook for Systematic Reviews of Interventions. 2011.

14. Bhandari M, Audige L, Ellis T, et al. Evidence-Based Orthopaedic Trauma Working Group. Operative treatment of extra-articular proximal tibial fractures. J Orthop Trauma. 2003;17(8):591-595.

15. Kurylo JC, Tornetta P. Extra-articular proximal tibial fractures: nail or plate? Instr Course Lect. 2013;62:61-77.

16. Mueller CA, Eingartner C, Schreitmueller E, et al. Primary stability of various forms of osteosynthesis in the treatment of fractures of the proximal tibia. J Bone Joint Surg Br. 2005;87(3):426-432.
17. Hansen M, Mehler D, Hessmann MH, et al. Intramedullary stabilization of extraarticular proximal tibial fractures: a biomechanical comparison of intramedullary and extramedullary implants including a new proximal tibia nail (PTN). J Orthop Trauma. 2007;21(10):701-709.

18. Lee SM, Oh CW, Oh JK, et al. Biomechanical analysis of operative methods in the treatment of extra-articular fracture of the proximal tibia. Clin Orthop Surg. 2014;6(3):312-317.

19. Schütz M, Kääb MJ, Haas N. Stabilization of proximal tibial fractures with the LIS-System: early clinical experience in Berlin. Injury. 2003;34(Suppl 1):A30-A35.

20. Messmer P, Regazzoni P, Gross T. New stabilization techniques for fixation of proximal tibial fractures (LISS/LCP). Ther Umsch. 2003; 60(12):762-767.

21. Oh JK, Oh CW, Jeon IH, et al. Percutaneous plate stabilization of proximal tibial fractures. $J$ Trauma. 2005;59(2):431-437.

22. Gaebler C, Berger U, Schandelmaier P, et al. Rates and odds ratios for complications in closed and open tibial fractures treated with unreamed, small diameter tibial nails: a multicenter analysis of 467 cases. J Orthop Trauma. 2001;15(6):415-423. 